\title{
Ubicación óptima de generación distribuida en sistemas de distribución usando un modelo de programación no lineal entero mixto
}

\section{Optimal location of distributed generation in distribution systems using a model of nonlineal whole mixed programming}

\section{Jesús María López Lezama}

Ingeniero electricista, doctor en Ingeniería Eléctrica. Docente de la Universidad de Antioquia. Medellín, Colombia.lezama@udea.edu.co

Clasificación del artículo: Investigación (Conciencias)

Fecha de recepción: 4 de junio de 2011

Fecha de aceptación: 29 de agosto de 2011

Palabras clave: Generación distribuida, programación no lineal entera mixta, sistemas de distribución.

Key words: Distributed generation, nonlinear whole mixed programming, distribution systems.

\section{RESUMEN}

En este artículo se presenta un modelo de programación matemática para la ubicación óptima de generación distribuida en sistemas de distribución. Se considera una empresa de distribución que puede comprar energía del mercado mayorista, y alternativamente puede instalar generación distribuida en su red. El modelo propuesto consiste en un problema de programación no lineal enero mixto, el cual es resuelto usando un software de optimización comercial. El modelo permite valorar de forma implícita el impacto de la generación distribuida y utilizar esta valoración para guiar las decisiones de inversión. Para mostrar la efectividad del modelo se realizan pruebas en un sistema de distribución de 34 barras, considerando diferentes precios de energía en el mercado mayorista. Los resultados muestran que la generación distribuida, cuando se ubica de manera óptima, puede contribuir al mejoramiento del perfil de tensiones y a la reducción de pérdidas.

\section{ABSTRACT}

This paper presents a mathematical programming model for optimal location of distributed generation in distribution systems. A distribution company is considered when it can buy power wholesale market, and alternatively, you can install distributed generation network. The proposed model is a nonlinear programming problem which is mixed in January solved using commercial optimization software. The model implicitly allows assessment of the impact of distributed generation and uses this assessment to guide investment decisions. To 


\section{con-ciencias}

show the effectiveness of the model is tested on a distribution of 34 bars considering different energy prices in the wholesale market. The results show that distributed generation, when it placed in an optimum manner, can contribute to improving the voltage profile and reduce losses.

\section{INTRODUCCIÓN}

La generación distribuida (GD) puede ser definida como la producción de electricidad a pequeña escala realizada cerca de los consumidores o en la red de distribución [1]. Actualmente, existe una gran variedad de tecnologías de GD, destacándose entre ellas las basadas en recursos renovables como generación eólica, fotovoltaica y generación con biomasa. Por otro lado, existen también tecnologías de GD basadas en recursos no renovables, entre las cuales se encuentran las microturbinas a gas, máquinas de combustión interna, etc. Aunque en la actualidad la mayoría de las tecnologías de GD no pueden competir directamente con la generación centralizada, es bien sabido que la GD puede traer beneficios como reducción de pérdidas técnicas, mejoramiento del perfil de tensiones y alivio de congestión [2]. Las ventajas económicas de la GD se pueden poner de manifiesto cuando ésta es utilizada en los horarios de demanda máxima, en los cuales los precios de la energía son mayores. En cuanto a las ventajas técnicas, pueden ser muy variadas como las ya mencionadas, y dependen principalmente de la ubicación y dimensionamiento de las unidades de GD en la red.

Con el creciente auge de la GD las empresas distribuidoras necesitan incorporar nuevas herramientas de planeación para sus redes. Tradicionalmente, los estudios de planeamiento en distribución se han basado en la ampliación de la capacidad de las subestaciones, la instalación de nuevas subestaciones, el refuerzo o construcción de nuevas líneas y la reconfiguración de redes [3][4]. No obstante, en la última década se han reportado numerosos trabajos que incluyen la ubicación de nuevas unidades de GD como una alternativa dentro de los estudios de planeamiento de redes de distribución. En [5] se presentan métodos analíticos para la ubicación óptima de GD considerando diferentes distribuciones de carga. En [6] se utilizan reglas que normalmente son aplicadas para la ubicación de condensadores como estrategia para ubicar GD en redes de distribución. $\mathrm{Si}$ dicha metodología es fácil de implementar, su principal desventaja radica en que solo puede ser aplicada en alimentadores con distribución de carga uniforme, lo cual limita considerablemente su aplicación. Dado que el problema de ubicación óptima de GD es intrínsecamente no lineal y no convexo, este problema es comúnmente abordado usando técnicas de optimización metaheurísticas como los Algoritmos genéticos [7], Búsqueda Tabú [8] y Partículas Swarm [9]. El objetivo de este artículo es contribuir en esta línea de investigación, para lo cual se propone un modelo de programación no lineal entero mixto, que permite encontrar la ubicación óptima de varias unidades de GD en la red. Dicho modelo considera un escenario en el cual la compañía distribuida puede suplir la demanda de su red, bien sea comprando energía del mercado mayorista, o instalando nuevas unidades de GD. Para reducir la complejidad computacional del problema, y facilitar el uso de software de optimización comercial, se limitan los casos de estudio a redes con estructura radial y se utiliza una versión simplificada de las ecuaciones balance de potencia.

\section{METODOLOGÍA}

El problema de ubicación óptima de GD propuesto en este artículo consiste en un modelo de programación no lineal entero mixto. Este tipo 


\section{con-ciencias}

de modelos son intrínsecamente no convexos y en la actualidad no existe un método de solución que garantice la obtención de un óptimo global. A continuación se describen las hipótesis del modelo y su formulación.

\subsection{Hipótesis iniciales}

- A diferencia de un modelo AC tradicional no se representan los desfases de la tensión en las barras de la red, ni tampoco se tiene en cuenta la potencia reactiva. Esta simplificación es habitual cuando se analizan redes de distribución radiales [10], [11].

- La compañía distribuidora participa en el mercado eléctrico donde puede comprar energía. De esta forma, la decisión de instalar GD está fuertemente influenciada por el precio de energía en el mercado mayorista.

- La demanda de energía y el precio de ésta en el mercado eléctrico son conocidos.

- No se modela la incertidumbre de las fuentes de energía, es decir, se consideran solamente tecnologías de GD despachables.

- Las unidades de generación distribuida pertenecen, en su totalidad, a la compañía distribuidora y solo se puede instalar una unidad en cada nodo del sistema.

- La red de distribución se alimenta de la red principal a través de una única subestación. El precio de la energía visto en la subestación es igual al precio de la energía del mercado mayorista.

\subsection{Aproximaciones del modelo de red}

Para reducir la complejidad del modelo se consideran algunas simplificaciones a las ecuaciones de balance de potencia. Estas simplificaciones son similares a las propuestas en [10] y son válidas para sistemas de distribución radiales y con una relación alta de $\mathrm{R} / \mathrm{X}$. La principal ventaja de estas simplificaciones consiste en que solo son consid- eradas la magnitud de las tensiones y la potencia activa, lo cual evita utilizar los ángulos y la potencia reactiva como variables de decisión.

En un modelo AC tradicional el flujo de potencia activa en la línea que conecta los nodos $n, m$ se puede expresar como lo indica la Ec. (1).

$$
\begin{gathered}
P_{m n}=\left|V_{n}\right|^{2} g_{n m}-\left|V_{n}\right|\left|V_{m}\right| g_{n m} \cos \left(\delta_{n m}\right)- \\
\left|V_{n}\right|\left|V_{m}\right| b_{n m} \operatorname{sen}\left(\delta_{n m}\right) ; \quad \forall 1_{m n} \in L
\end{gathered}
$$

Donde;

$P_{m m}$ : flujo de potencia en la línea que conecta los nodos $n, m$

$V_{n}$ : magnitud de la tensión en la barra $n$

Considerando la diferencia angular entre barras adyacentes como aproximadamente igual a cero se tiene la expresión dada por la Ec. (2).

$$
\begin{gathered}
P_{m n}=\left|V_{n}\right|^{2} g_{n m}-\left|V_{n}\right|\left|V_{m}\right| g_{n m}= \\
g_{n m}\left|V_{n}\right|\left(\left|V_{n}\right|-\left|V_{m}\right|\right) ; \quad \forall 1_{m n} \in L
\end{gathered}
$$

Considerando que $g_{n m}=R_{n m} / Z_{n m}^{2}$ la Ec. (2) puede ser reescrita como ilustra en (3).

$$
P_{n m}=\frac{R_{n m}\left|V_{n}\right| \cdot\left(\left|V_{n}\right|-\left|V_{m}\right|\right)}{Z_{n m}^{2}}
$$

Donde;

$Z_{m m}:$ impedancia de la línea que conecta los nodos $n, m$

Las pérdidas de potencia se pueden expresar como la suma de los flujos en ambas direcciones como se muestra en la Ec. (4).

$$
P_{n m}^{\text {loss }}=P_{n m}+P_{m n}
$$

Remplazando (3) en (4) y simplificando, las pérdidas de potencia se pueden escribir como se muestra en (5). 


\section{con-ciencias}

$$
P_{n m}^{l o s s}=\frac{R_{n m}\left(\left|V_{n}\right|-\left|V_{m}\right|\right)^{2}}{Z_{n m}^{2}}
$$

\subsection{Restricciones}

\subsubsection{Balance de potencia}

Teniendo en cuenta las simplificaciones indicadas en (1) - (5) y al considerar una única subestación conectada en el nodo 1, la restricción de balance de potencia activa en la subestación está dada como se indica en la Ec. (6). Este balance considera la potencia comprada en el mercado eléctrico a través de la subestación, la demanda en este nodo, el posible racionamiento y los flujos a través de las líneas que conectan la subestación con sus barras adyacentes, al igual que sus respectivas pérdidas.

$$
\begin{gathered}
P_{S E}+P_{R 1}-P_{D 1}-\sum_{\substack{m \in \Omega 1 \\
m>1}} \frac{R_{1 m}\left(\left|V_{1}\right|-\left|V_{m}\right|\right)^{2}}{Z_{1 m}^{2}}+ \\
\sum_{\substack{n \in \Omega 1 \\
m>1}} \frac{R_{1 m}\left|V_{1}\right| \cdot\left(\left|V_{1}\right|-\left|V_{m}\right|\right)}{Z_{1 m}^{2}}=0
\end{gathered}
$$

Donde;

$P_{S E}$ : potencia entregada a través de la subestación

$P_{R i}$ : racionamiento en la barra $i$

$P_{D i}:$ potencia demandada en la barra $i$

De forma análoga, se puede generalizar el balance de potencias, para los nodos diferentes a la subestación, mediante la Ec. (7). Note que a diferencia de la Ec. (6) en la Ec. (7) se considera la presencia de generación distribuida. La GD, en este caso, está asociada con la variable binaria $x_{G D n j}$, la cual, si vale 1 significa que hay una unidad de GD ubicada en este nodo, en caso contrario (si vale 0 ), indica que no hay generación distribuida.

$$
\sum_{\substack{m \in \Omega n \\ m>n}} \frac{R_{n m}\left|V_{n}\right| \cdot\left(\left|V_{n}\right|-\left|V_{m}\right|\right)}{Z_{n m}^{2}}+\sum_{\substack{m \in \Omega n \\ m<n}} \frac{R_{n m}\left|V_{m}\right| \cdot\left(\left|V_{m}\right|-\left|V_{n}\right|\right)}{Z_{n m}^{2}}-
$$

$$
\sum_{m \in \Omega n} \frac{R_{n m}\left(\left|V_{n}\right|-\left|V_{m}\right|\right)^{2}}{Z_{n m}^{2}}+x_{G D n j} P_{G D j}-P_{D n}+P_{R n}=0 \quad \forall n>1
$$

Donde;

$\Omega n$ : conjunto de nodos conectados al nodo $m$

$x_{G D n j}$ : variable binaria que indica la existencia de generación distribuida en el nodo $n$

$P_{G D j}:$ potencia entregada por la unidad de generación distribuida $j$

\subsubsection{Límites de flujo de potencia}

La introducción de GD en la red de distribución puede dar lugar a flujos de potencia bidireccionales, por tanto, se tienen en cuenta los límites de las líneas en ambas direcciones como se indica en la Ec. (8).

$$
-P_{n m}^{M a x} \leq \frac{R_{n m}\left|V_{n}\right| \cdot\left(\left|V_{n}\right|-\left|V_{m}\right|\right)}{Z_{n m}^{2}} \leq P_{n m}^{M a x}
$$

Donde;

$P_{n m}^{M a x}$ : límite máximo del flujo de potencia en la línea que conecta los nodos $n, m$

\subsubsection{Límites de tensión en las barras}

La magnitud de la tensión en las barras del sistema de distribución tiene un límite máximo y un límite mínimo, como establece la Ec. (9).

$$
\left|V_{n}\right|^{\text {Min }} \leq\left|V_{n}\right| \leq\left|V_{n}\right|^{\text {Max }} ; \quad \forall n \in N
$$

Donde;

$V_{n}^{M i n}: \quad$ límite mínimo de la magnitud de la tensión en la barra $n$

$V_{n}^{M a x}$ : límite máximo de la magnitud de la tensión en la barra $n$

$N \quad$ : conjunto de nodos 


\subsubsection{Límites de potencia suministrada a través de la subestación}

La Ec. (10) ilustra los límites mínimos y máximos de la potencia suministrada a través de la subestación. Esta ecuación representa las restricciones técnicas de capacidad de la subestación, las cuales limitan a su vez la potencia que puede ser comprada en el mercado mayorista.

$$
P_{S E}^{M i n} \leq P_{S E} \leq P_{S E}^{M a x}
$$

Donde;

$P_{S i n}^{\text {Min }}$ potencia mínima entregada a través de

$P_{S E} \quad$ la subestación

$P_{S E}^{M a x}$ : potencia máxima entregada a través de

\subsubsection{Límites de producción de la generación distribuida}

La Ec. (11) establece la restricción de límites mínimos y máximos de la potencia suministrada por las unidades de GD.

$$
P_{G D j}^{M i n} \leq P_{G D j} \leq P_{G D j}^{M a x} ; \quad \forall j \in J
$$

Donde; $P_{G D j}^{M i n} \quad$ : potencia mínima de la unidad de genera-
$\quad$ ción distribuida $j$

$P_{G D j}^{M a x}:$ potencia máxima de la unidad de generación distribuida $j$

$J \quad$ : conjunto de unidades de generación distribuida

\subsubsection{Restricciones de deslastre de carga}

Para cada nodo de carga se asocia una variable que representa el deslastre de carga o racionamiento de energía. Este deslastre de carga debe ser positivo y menor que la demanda del nodo, como lo indica la Ec. (12).

$$
0 \leq P_{R i} \leq P_{D i} ; \quad \forall i \in K
$$

Donde;

$K$ : conjunto de barras donde hay racionamiento de energía

\subsubsection{Restricción de variable binaria para ubi- car las unidades de GD}

La Ec. (13) corresponde a la variable binaria de decisión sobre ubicar (si vale 1) o no (si vale 0 ) una unidad de GD en una determinada barra.

$$
x_{G D n j} \in\{0,1\} ; \quad \forall j \in J ; \forall n \in N
$$

\subsubsection{Restricción de número de unidades de GD a ubicar en la red}

En el modelo propuesto, el número máximo de unidades de GD para ser instalados en la red es determinado a priori y está dado por la Ec. (14). Además solo se permite instalar una unidad de GD por nodo como lo indicado en la Ec. (15).

$$
\begin{gathered}
\sum_{n \in N} \sum_{j \in J} x_{D G n j} \leq N_{D G}^{M a x} \\
\sum_{n \in N} \mu_{n j} \leq 1
\end{gathered}
$$

Donde;

$N_{D G}^{M a x}$ : número máximo de unidades de GD a ubicar en la red.

\subsection{Función objetivo}

La función objetivo propuesta para el modelo de ubicación óptima de generación distribuida está compuesta por cuatro términos como se ilustra en la Ec. (16).

$$
\underset{x_{G D j}, P_{S E}, P_{G D j}, P_{R i}, V_{n}}{\operatorname{Min}} \sum_{j \in J} C I_{G D j} P_{G D j}^{M a x} x_{G D j}+
$$




\section{con-ciencias}

$$
\sum_{j \in J} C O_{G D j} P_{G D j} x_{G D j}+C_{S E} P_{S E}+\sum_{i \in K} C R_{i} P_{R i}
$$

Donde;

$C I_{G D j}:$ costo de inversión de la unidad de gene
ración distribuida $j$

$C O_{G D j}$ : costo de operación de la unidad de gene ración distribuida $j$

$C R \quad$ : costo de racionamiento

En este caso se pretende la minimización de los costos en los que incurre la empresa distribuidora. El primer término representa el costo de inversión en GD. Este término corresponde al costo de adquirir e instalar la unidad de GD. Tal costo depende de la capacidad y tipo de tecnología y su rango de variación es muy extenso, siendo típicamente mayor en energías renovables. Este costo se expresa como costo horario (U\$/h). El segundo término corresponde al costo de operación de la unidad de GD y se expresa también como costo horario. El tercer término corresponde al costo de la potencia comprada en el mercado mayorista y suministrada a través de la subestación. Al ser expresado como costo horario, corresponde al costo de la energía. El último término corresponde al costo de la potencia no suministrada. Este costo representa las penalizaciones en las que puede incurrir la empresa distribuidora al no suministrar la energía a los usuarios y suele ser mucho mayor que los otros tres costos ya descritos.

\section{RESULTADOS}

Para validar la aplicabilidad del modelo propuesto se utiliza el sistema de distribución de 34 barras presentado en la Fig. 1. Los datos de línea de este sistema pueden ser consultados en [11]. La Fig. 2 ilustra la distribución de carga para una demanda media de $12 \mathrm{MW}$. Se puede observar que gran parte de la demanda está ubicada en las últi-

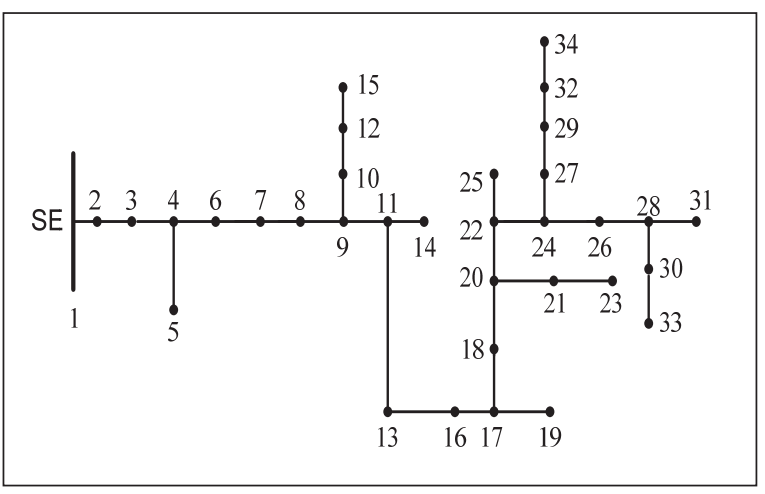

Fig. 1. Sistema de distribución de 34 barras.

mas barras, lejos de la subestación. En este caso se pretenden ubicar varias unidades de GD a gas, cada una con capacidad de 1.0MW. Se consideran costos de inversión de U\$500.000 por MW instalado y costos de operación de $60 \mathrm{U} \$ / \mathrm{MWh}$ [10], [12]. Considerando un financiamiento de las unidades a 10 años, con un interés anual del $9 \%$, el costo de inversión horario por cada unidad es U\$ 8,9. El costo de la energía en el mercado mayorista se considera igual a $65,0 \mathrm{U} \$ / \mathrm{MWh}$ y el costo de racionamiento de 200U\$/MWh. Para la subestación se considera una capacidad mínima de 0MW y máxima de 14MW. Los límites mínimos y máximos de tensión son 0,95 y 1,05 p.u, respectivamente. El modelo propuesto se implementó en GAMS [13].

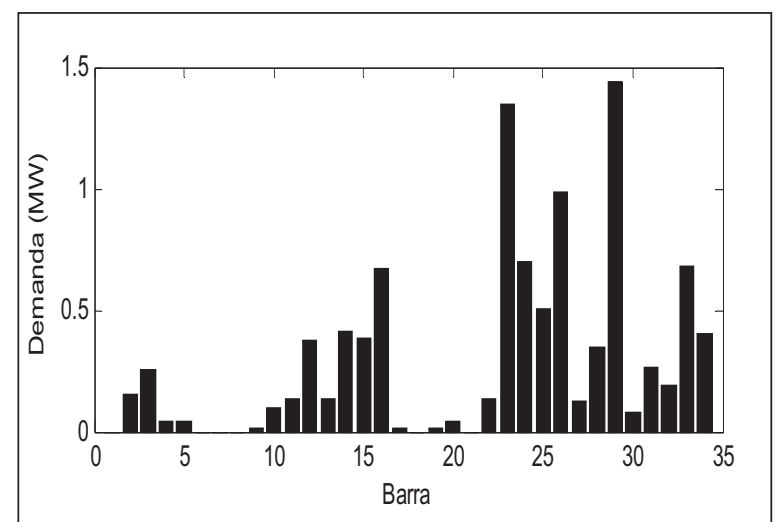

Fig. 2. Distribución de la demanda del sistema de distribución de la Fig. 1. 


\section{con-ciencias}

\subsection{Operación sin generación distribuida}

Inicialmente se hacen pruebas en el sistema sin GD. Para ello se resuelve el modelo propuesto considerando que toda la energía del sistema es suministrada a través de la subestación. Esto se hace fijando las variables binarias en cero. La Fig. 3 ilustra los precios marginales en cada una de las barras del sistema. Por definición, estos precios corresponden a las variables duales de las restricciones de balance de potencia (Ec. (6) y (7)) y representan el costo de suministrar un MW adicional en una barra dada del sistema [14]. En la Fig. 3 se puede observar que el precio marginal en la barra 1 es de U\$65 mientras el precio en las últimas barras es cercano a U\$78. Esto significa que es mucho más costoso suministrar energía en las últimas barras que en las barras cercanas a la subestación. Dado que no se presenta deslastre de carga y que no hay ninguna restricción activa, la diferencia en los precios nodales se debe completamente a las pérdidas de potencia activa. Para suministrar un MW adicional a las barras más alejadas de la subestación se incurre en pérdidas mayores que para suministrarlo en las barras más cercanas a la subestación. De hecho, suministrar un MW adicional en la barra de la subestación tendrá un costo de U\$65, sin embargo, suministrar este mismo MW al nodo 20 tiene un costo de U\$76,9. Esto significa que una unidad de GD que pueda proveer energía en este nodo a un costo menor que U\$76,9 le representaría ahorros a la compañía distribuidora. Esta observación permite concluir que la rentabilidad de la GD no está condicionada a que el costo de su energía sea menor al precio del mercado mayorista, sino más bien, a que sea menor al costo marginal de la energía de la barra en la que esté ubicada.

La Fig. 4 ilustra el perfil de tensiones de la red ajustando el voltaje en la subestación en 1,05 p.u. Se puede observar que a pesar de que las tensiones están dentro del rango permisible, existe una importante caída de tensión a lo largo de los alimentadores.

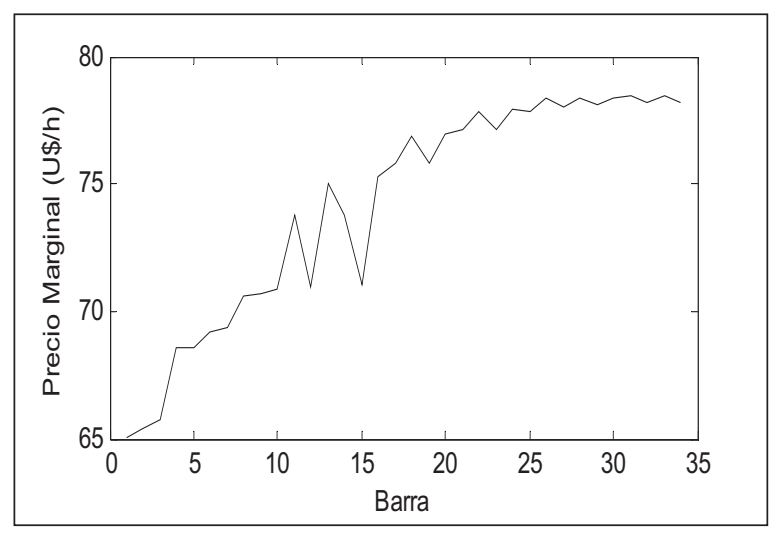

Fig. 3. Precios marginales del sistema sin GD.

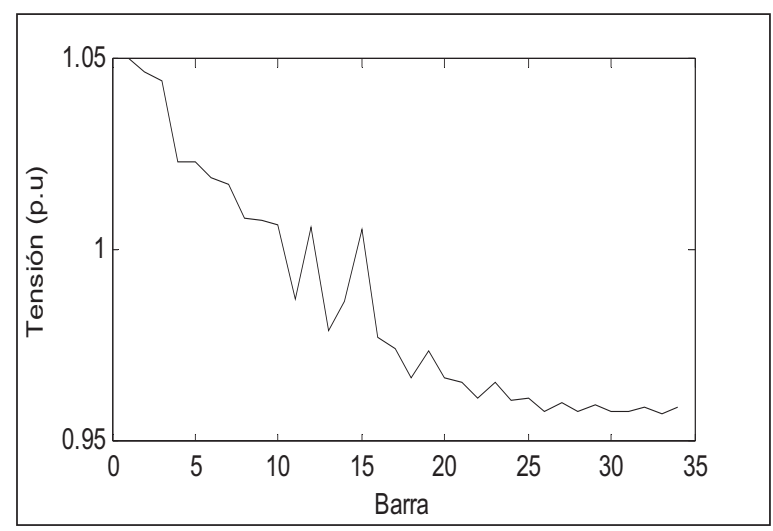

Fig. 4. Perfil de tensiones del sistema sin GD.

\subsection{Operación con generación distribuida}

Para resolver el modelo propuesto se considera un número máximo de 6 unidades de GD de $1 \mathrm{MW}$ a ser instaladas en la red. Este número está dado por el máximo porcentaje de penetración de GD que se quiera permitir en la red. En este caso limitamos arbitrariamente este valor a un $50 \%$. Adicionalmente, se considera que solo se puede instalar una unidad de GD por nodo y que todos los nodos son aptos para recibir GD.

Al resolver el problema de optimización se encuentra que para minimizar los pagos de la compañía distribuidora, lo más rentable es instalar solamente 4 unidades de GD en las barras 23, 24, 39 


\section{con-ciencias}

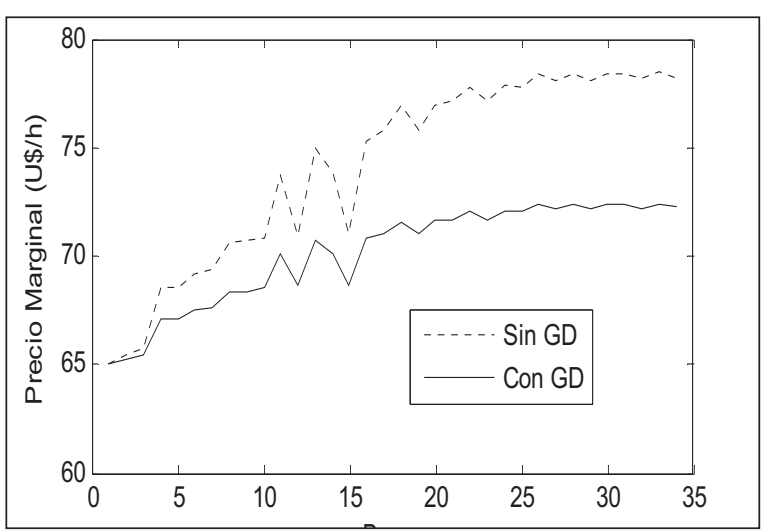

Fig. 5. Precios marginales del sistema con y sin GD.

y 33. Los nuevos precios marginales del sistema, tras instalar las unidades de GD, se ilustran en la Fig. 5. Se puede observar que la GD contribuye significativamente a la reducción de los precios marginales. En este caso, suministrar un MW adicional a los últimos nodos de la red tiene un costo ligeramente menor a U\$70. Esto ocurre porque la demanda líquida de la red disminuye, lo que contribuye a la reducción de las pérdidas en la red, las cuales son las responsables de las diferencias en los precios nodales. La Fig. 6 ilustra el perfil de tensiones de la red con y sin GD. Se puede observar que al instalar GD el perfil de tensiones mejora de forma considerable. En este caso, al ajustar la tensión de la subestación en 1,05 p.u, la tensión en las últimas barras es apenas ligeramente menor a 1,00 p.u.

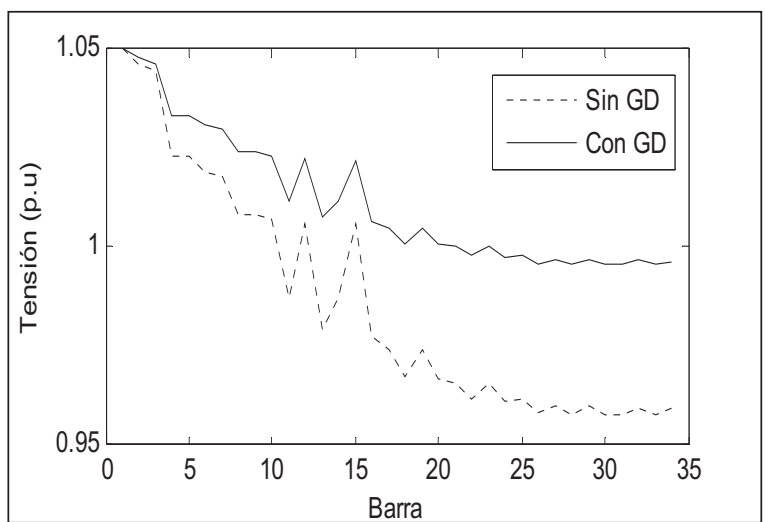

Fig. 6. Perfil de tensiones con y sin GD.
Tabla 1. Datos de la red con y sin generación distribuida.

\begin{tabular}{|l|c|c|c|}
\hline & $\begin{array}{c}\text { Pagos } \\
\mathbf{( U \$ / \mathbf { h } )}\end{array}$ & $\begin{array}{c}\text { Pérdidas } \\
(\%)\end{array}$ & $\begin{array}{c}\text { Tensión } \\
\text { mínima (p.u) }\end{array}$ \\
\hline Sin GD & 841,752 & 7,3359 & 0,9575 \\
\hline Con GD & 749,035 & 3,1477 & 0,9955 \\
\hline Diferencia & 92,717 & 4,1882 & $-0,0380$ \\
\hline
\end{tabular}

En la tabla 1 se resumen los impactos de la GD en la red. Note que si se suman los costos de inversión y operación de la GD se tiene un costo total horario de 68,9U\$/MWh, el cual es mayor al costo de la energía en el mercado mayorista $(65,0 \mathrm{U} \$$ / MWh). Aun así, la GD le ahorra a la compañía distribuidora una cantidad importante de dinero. En cuanto a las pérdidas activas, se encontró que la reducción de éstas cuando se instalan estratégicamente las unidades de GD es superior al 50\%.

En la tabla 2 se ilustra la ubicación de las unidades de GD considerando diferentes precios del mercado mayorista. Se puede observar que instalar GD es rentable para la distribuidora, inclusive cuando el precio de la energía en el mercado mayorista es un poco menor al costo total de inversión y operación de la GD. Por otro lado, si el precio del mercado mayorista es mucho menor al costo de inversión y operación de las unidades de GD no es rentable instalar dichas unidades como se puede observar para el caso de $55 \mathrm{U \$} /$ MWh.

Tabla 2. Ubicación de GD considerando diferentes precios del mercado mayorista.

\begin{tabular}{|c|c|c|}
\hline $\begin{array}{c}\text { Precio de energía } \\
\text { (U\$/MWh) }\end{array}$ & $\begin{array}{c}\text { Número } \\
\text { de unidades }\end{array}$ & Ubicación \\
\hline 55 & 0 & -- \\
\hline 60 & 2 & 29,33 \\
\hline 65 & 4 & $23,24,29,33$ \\
\hline 70 & 5 & $16,23,26,29,33$ \\
\hline
\end{tabular}




\section{con-ciencias}

\section{CONCLUSIONES}

En este artículo se presentó un modelo de programación no lineal entero mixto para la ubicación óptima de generación distribuida en sistemas de distribución. Los resultados sobre un sistema de prueba de 34 barras indicaron que la mejor ubicación de las unidades corresponde a los nodos más alejados de la subestación y con mayor demanda. Se pudo observar también que el número óptimo de unidades de GD por ubicar depende de la dife- rencia entre el costo de la energía en el mercado mayorista y el costo total de producción de la GD. Los resultados muestran que la ubicación estratégica de las unidades de GD permite mejorar el perfil de tensiones, reducir las pérdidas y puede llegar a representar importantes ahorros para la distribuidora en la compra de energía. En un trabajo futuro se tendrá en cuenta la variabilidad de la demanda y la posibilidad de incluir otro tipo de tecnologías de GD.

\section{REFERENCIAS}

[1] T. Ackermann, G. Andersson and L. Soder, "Distributed generation: a definition," Electric Power Systems Research, vol. 71, pp. 119-128, Oct. 2004.

[2] L. Ochoa, A. Padilha-Feltrin and G.P. Harrison, "Evaluating distributed generation impacts with a multiobjective index," IEEE Transactions on Power Delivery, vol. 21, no. 3, pp. 1452-1458, Jul. 2006.

[3] E. Miguez, J. Cidras, E. Diaz-Dorado, and J.L. Garcia-Dornelas, "An improved branch Exchange algorithm for large scale distribution planning," IEEE Transactions on Power Systems. vol. 17, no. 4, pp. 931936, Aug. 2002.

[4] T. Asakura, T. Genji, T. Yura, N. Hayashi, and Y. Fukuyama, "Long-term distribution network expansion planning by network reconfiguration and generation of constructions plans," IEEE Transactions on Power Systems, vol. 18 no. 3, pp. 1196-1204, Sep. 2003.

[5] C. Wang and M. Hashen, "Analytical approaches for optimal placement of distributed generation sources in power systems," IEEE Transactions on Power
Systems, vol. 19, no. 4, pp. 2068-20-76, Nov. 2004.

[6] H. Willis, "Analytical methods and rules of thumb for modeling DG distribution interaction," in Proc. 2000 IEEE Power Engineering Society Summer Meeting. Seattle WA, vol. 3 pp. 1643-1644, July 2000.

[7] G. Celli, E. Ghiani, S. Mocci, and F. Pilo, "A multiobjective evolutionary algorithm for the sizing and sitting of distributed generation," IEEE Transactions on Power Systems, vol. 20, no. 2, pp. 750-757, Nov. 2005.

[8] R.S. Marciel and A. Padilha-Feltrin, "Distributed generation impact evaluation using a multi-objective Tabu Search," 15th International Conference on Intelligent System Applications to Power Systems, ISAP, Curitiba, 2009.

[9] Y. Alinejad-Beromi, M. Sedighizadeh, and M. Sadighi, "A particle swarm optimization for sitting and sizing of distributed generation in distribution network to improve voltage profile and reduce THD and losses," Proceedings of the 43th International Universities Power Engineering Conference, Padova, Jul. 2008. 


\section{con-ciencias}

[10] W. El Khattam, Y. Hegazy and M.M.A. Salama, "Optimal investment planning for distributed generation in a competitive electricity market," IEEE Transactions on Power Systems, vol. 9, no. 3, pp. 16741684, Aug. 2004.

[11] J. Lopez, A. Padilha, J. Contreras and J.I. Muñoz, "Optimal contract pricing of distributed generation in distribution networks," IEEE Transactions on Power Systems, vol. 26, no. 1, pp. 128-136, Feb. 2011.
[12] E. Silva and J. Haddad, Geração Distribuída: Aspetos Técnicos, Ambientais e Institucionais. Rio de Janeiro: Editora Interciencia, 2006.

[13] The GAMS Development Corporation Website, 2011. [En línea] Disponible en: http://www.gams.com

[14] F. Schweppe, M. Caramanis, R. Tabors and R.E. Bohn, Spot Pricing of Electricity, Boston: Kluwer Academic Publishers 1988. 